\title{
La tradición de jardinería del siglo XVIII y la posibilidad de emitir juicios estéticos puros de objetos artísticos
}

\author{
RICARDO ROJAS \\ Pontificia Universidad Católica del Perú \\ https://doi.org/I0.I8800/estudiosdefilosofia.201701.004
}

Resumen: En el tercer momento de la "Analítica de lo bello" de la Crítica de la facultad de Juzgar, Kant establece la diferencia entre juicios puros de gusto y juicios de belleza adherente. A juicio del autor, las definiciones presentadas resultan problemáticas a la hora de intentar conciliarlas con juicios de belleza artística. En principio, toda obra de arte supone conceptos y contenidos que la determinan como objeto artístico, por lo que no sería posible emitir juicios puros de gusto sobre ellos. Es necesario articular las ideas en relación a la naturaleza de la producción artística presentadas en las secciones sobre arte bello y el genio, donde se introduce el concepto de "ideas estéticas" (entendidas como intuiciones internas de los objetos artísticos), para superar aquellas dificultades. Por último, el debate sobre paisaje y jardinería en el siglo XVIII permite entender cómo se puede emitir juicios estéticos puros a partir de dos formas distintas de presentar ideas estéticas en un género artístico.

Palabras clave: juicios puros de gusto, arte bello, genio, ideas estéticas, jardinería en el siglo XVIII.

Abstract: "18th Century Gardening Tradition, and the Possibility of Pure Aesthetic Judgments on Artistic Objects". At the third section of the "Analytic of the Beautiful" of the Critique of Judgement, Kant establishes the difference between pure judgements of taste and judgements of adherent beauty. The Author contends that the definitions presented there are problematic when one attempts to reconcile them with judgements of artistic beauty. In principle, every work of art supposes certain concepts and contents that determine it as an artistic object, so it would not be possible to formulate pure judgements of taste in their regard. In order to overcome these difficulties, it becomes necessary to articulate the ideas in relation to the nature of artistic production that Kant presents in the sections on Fine Art and the Genius, where the 
concept of "aesthetic ideas" (understood as internal intuitions of artistic objects) is introduced. Finally, the debate on landscape and gardening in the $18^{\text {th }}$ century allows us to understand how pure judgements of taste can be made from two different ways of presenting aesthetic ideas in an artistic genre.

Keywords: pure judgements of taste, Fine Art, Genius, aesthetic ideas, gardening in the $18^{\text {th }}$ century.

\section{§ 1. Introducción}

Del tercer momento de la "Analítica de lo bello", que desarrolla la relación entre el sujeto que juzga y el objeto (o su representación) que es juzgado, expuesta en la Crítica de la facultad de juzgar de Kant, se desprenden algunas conclusiones problemáticas. Estas afectarán a varios pasajes de la tercera Crítica. El presente texto se centra en una en particular: la aparente imposibilidad de emitir juicios puros de gusto de objetos artísticos.

Por un lado, en contraste con los juicios puros de gusto (enjuiciamientos de una belleza libre), Kant establece enjuiciamientos de belleza adherente. Estos son juicios estéticos que suponen ciertos contenidos o conceptos del objeto en el juicio, si bien no se establecen como fundamento de determinación de este. Por otro lado, Kant sostiene que los juicios puros de gusto reposan únicamente en un principio de conformidad a fin formal de las facultades de conocimiento, y son independientes de contenidos y conceptos que determinen al objeto (o a su representación).

El problema surge al intentar conciliar los juicios de belleza artística con las definiciones brindadas en el tercer momento de la "Analítica de lo bello". En última instancia, las obras de arte suponen un concepto de "arte" que las determina como tales, por lo que, a partir de lo dicho en la "Analítica", no sería posible emitir un juicio puro de gusto sobre objetos artísticos. Es necesario recurrir a las secciones acerca del arte bello y del genio, en donde se explora la naturaleza de la producción artística, para intentar demostrar que es posible emitir juicios puros de gusto de obras de arte. La presentación de ideas estéticas en objetos artísticos (actividad propia del genio) y su posterior 
aprehensión en la contemplación de la obra, permitiría explicar la posibilidad de establecer juicios estéticos puros de belleza artística, ya que son entendidas como intuiciones internas de los objetos artísticos (por lo que ningún concepto puede serles enteramente adecuado). Además, la incorporación del debate sobre paisaje y jardinería entre la tradición francesa y la inglesa en el siglo XVIII sirve de ejemplo para entender cómo se puede emitir juicios estéticos puros a partir de dos formas específicas y radicalmente distintas de presentar ideas estéticas en un mismo género artístico.

\section{§ 2. Análisis de la Crítica de la facultad juzgar}

\section{$\S 2.1$. Consideraciones sobre el tercer momento}

Al finalizar el tercer momento de la "Analítica de lo bello", Kant proporciona la siguiente definición de belleza: "Belleza es forma de la conformidad a fin de un objeto, en la medida en que ésta sea percibida en éste sin representación de un fin" (I99I, §17, B6I). La definición brindada por Kant se deduce del principio de conformidad a fin formal (una conformidad a fin sin fin), desarrollado ampliamente en esta sección.

Kant determina que el juicio de gusto no puede reposar sobre ningún fin meramente subjetivo, debido a que tal juicio se sostendría en un interés que se produce por una complacencia en el objeto. Tampoco puede ser determinado por la representación de un fin objetivo, es decir, de algún concepto de lo bueno. Por el contario, los juicios de gusto se producen por una complacencia desinteresada (la cual es analizada por Kant en el primer momento de la "Analítica de lo Bello"). El juicio estético se fundamenta solamente en "la relación de las fuerzas representacionales entre sí, en cuanto son determinadas ellas por una representación" (I99I, §II, B34). Además, anteriormente se ha demostrado que el enjuiciamiento antecede al sentimiento de placer en el sujeto, por lo que "un agrado que acompaña a la representación no puede contener el fundamento de determinación del juicio” (I99I, §II, B35). Este sólo puede manifestarse a partir del libre juego de las facultades de conocimiento que juzgan, imaginación y entendimiento. El fundamento de un juicio de gusto no es otro que la forma como se relacionan ambas facultades cuando juzgan sobre un objeto dado, o sobre la representación de este: "Lo que constituye 
a la complacencia que, sin concepto, juzgamos universalmente comunicable $y$, con ello, al fundamento de determinación del juicio de gusto, no puede ser otra cosa que la conformidad a fin subjetiva en la representación de un objeto, sin fin alguno (ni objetivo ni subjetivo) y, consecuentemente, la mera forma de la conformidad a fin en la representación por la que nos es dado un objeto, en la medida que somos conscientes de aquélla" (I99I, §II, B35).

En efecto, el juicio estético no trae consigo ningún fin, pero es conforme a fin. Esto implica, a nivel puramente formal, que el enjuiciamiento reposa en la relación armónica de la imaginación y el entendimiento, "como si" fuesen conforme a fin: "podemos al menos observar una conformidad a fin según la forma, aun sin que pongamos en su fundamento un fin (como materia del nexus finalis), y advertirla en objetos, si bien no de otro modo más que por reflexión" (199I, §।0, B34). Esta relación se explica como un principio a priori que la facultad de juzgar se pone a sí misma, aquello que el filósofo llama una "conformidad a fin" estética o teleológica.

Por consiguiente, en el §I2 Kant se propone demostrar que el juicio de gusto reposa en fundamentos a priori. La conformidad a fin (sin fin), que es el aspecto formal de la relación entre las facultades al expresar un juicio estético, constituye, además, el principio a priori sobre el que reposa el juicio de gusto. La conformidad a fin formal de nuestras facultades consiste en una relación particular entre ellas: la de una concordancia armónica, o de libre juego, entre la imaginación y el entendimiento. Solo si esta relación formal específica se establece como fundamento de determinación del juicio es posible decir que se expresa un juicio de gusto puro. Por lo tanto, el placer es suscitado a partir de la conciencia de la actividad subjetiva del juego libre entre imaginación y entendimiento: "La conciencia de la conformidad a fin puramente formal en el juego de las fuerzas de conocimiento del sujeto, a propósito de una representación por la cual es dado un objeto, es el placer mismo, porque contiene un fundamento de determinación de la actividad del sujeto en vista de la vivificación de sus fuerzas de conocimiento $y$, por tanto, una causalidad interna (que es conforme a fin) con vistas al conocimiento en general, mas sin estar restringida a un conocimiento determinado, $y$, con ello, contiene una mera forma de conformidad a fin subjetiva de una representación en un juicio estético" (199I, §I2, B37). 
En conclusión, Kant afirma en el $\S \mid 2$ que el sentimiento de placer se suscita en el sujeto solamente si este fundamenta su juicio en una conformidad a fin formal de sus facultades. El sujeto se vuelve consciente de la vivificación de sus fuerzas: entendimiento e imaginación. De esto puede desprenderse que la pretensión de universalidad subjetiva, que Kant le atribuye al o analiza en el juicio de gusto en el segundo momento de la "Analítica de lo bello", consiste en suponer que cada uno es capaz de establecer una relación de conformidad a fin formal entre sus facultades de conocimiento. El juicio de gusto es la expresión de un sentimiento de placer fundamentado únicamente en la relación armónica que establecen las facultades de conocimiento con relación a una representación.

Para Kant, el placer en el juicio estético es aquí contemplativo, por lo que no despierta ningún interés por el objeto (1991, §12, B37). No obstante, afirma que tiene una causalidad: la de "conservar sin propósito ulterior el estado de la representación misma y la actividad de las facultades de conocimiento. Nos quedamos en la contemplación de lo bello, porque esta contemplación se refuerza y reproduce a sí misma (...)" (199I, §I2, B37). El sentimiento de placer es un estado representacional del sujeto en el que se busca conservar, sin ningún propósito, tanto la representación del objeto que suscita un juicio de gusto, como la actividad de las facultades de conocimiento a partir de un principio de conformidad a fin formal.

Sin embargo, la principal interrogante del tercer momento se encuentra hacia el final del $\S 12$. Si el juicio de gusto se fundamenta en la conformidad a fin formal de las facultades de conocimiento, iesto significa que solo se fundamenta en un principio formal y no según contenidos? ¿Acaso los conceptos e ideas que están contenidos en (o que se produzcan a partir de) la representación dada no pueden ser el fundamento de determinación a priori de los juicios de gusto, sino que este reposa únicamente en un principio de conformidad a fin meramente formal?

Tal es la línea a la que apunta Kant a partir del §|3, en donde busca separar el juicio de gusto de todo atractivo y emoción. En dicha sección afirma: "Todo interés corrompe el juicio del gusto y le quita su imparcialidad, sobre todo cuando no hace, como el interés de la razón, que la conformidad a fin anteceda al placer, sino que funda a aquélla en este: lo último ocurre siempre en el juicio 
estético acerca de algo en tanto que ello deleite o duela" (I99|, §|3, B38). El fundamento de determinación de los juicios de gusto es exclusivamente subjetivo, por lo que Kant afirma que es un error sostener juicios estéticos en afinidades atractivas o emocionales. Estas, bajo otro punto de vista, son objetivas e interesadas. Además, Kant introduce y define los juicios puros de gusto en este parágrafo, para diferenciarlos de juicios que son influidos por atractivos y emociones, señal de un gusto bárbaro: "Un juicio de gusto, sobre el cual no tengan influencia alguna el atractivo y la emoción (aunque puedan éstos unirse a la complacencia en lo bello), que posea pues como fundamento de determinación sólo la conformidad a fin de la forma es un juicio puro de gusto" (I99I, §I3, B38).

Esta diferenciación se esclarece en el §|4, en donde Kant divide los juicios estéticos en empíricos y puros. Dice al respecto: "Los primeros son los que, respecto de un objeto o del modo de representarlo, enuncian agrado o desagrado; los otros, los que [a este respecto] enuncian belleza; aquéllos son juicios de los sentidos (juicios estético materiales), y solo estos otros (en cuanto formales) juicios de gusto en propia acepción" (I99|, §|4, B39). Los juicios estéticos empíricos admiten una sensación como fundamento de determinación, mientras que un juicio puro de gusto "no tiene por fundamento de determinación ni un atractivo ni una emoción, en una palabra, ninguna sensación, como materia del juicio estético" (199I, §|4, B43). Una vez más, Kant insiste en un principio formal como fundamento de los juicios estéticos puros, en contraste con aquellos juicios sobre la belleza que se sostienen en meros atractivos.

En el $§ \mid 5$, Kant sostiene que los juicios puros de gusto no pueden fundamentarse a partir de conceptos; específicamente, a partir de un principio de conformidad a fin objetivo interno al objeto, que supone un concepto de perfección en éste. Para Kant, el criterio de perfección siempre trae a colación el aspecto objetivo de una representación, ya que no disocia la forma del contenido y centra su enjuiciamiento en el último. Por este camino, el objeto se vuelve bello debido a que el juicio se sostiene en un concepto de perfección en la representación. Sin embargo, Kant sostiene que lo formal en la representación de una cosa no da a conocer por sí mismo ninguna conformidad a fin objetiva, sino que solamente indica una cierta conformidad a fin del estado representacional en el sujeto, esto es, su disposición para aprehender una forma dada (I99I, §I5, 
B46). Los juicios estéticos no proporcionan ningún conocimiento del objeto, por lo que no pueden fundamentarse en conceptos o propiedades de este: "El juicio estético es único en su especie, que no da en absoluto un conocimiento (ni siquiera confuso) del objeto; esto último ocurre sólo a través de un juicio lógico, mientras que, por el contrario, aquél refiere la representación por la cual es dado un objeto únicamente al sujeto, sin que se haga advertir ninguna cualidad del objeto, sino sólo la forma conforme a fin en la determinación de las fuerzas representacionales que se ocupan de aquél. El juicio también se denomina estético, precisamente porque su fundamento de determinación no es un concepto, sino el sentimiento (del sentido interno) de ese acuerdo en el juego de las fuerzas del ánimo, en tanto que éstas son solamente sentidas" (1991, §15, B47).

Después de establecer que los juicios puros de gusto no se fundamentan ni en sensaciones ni en conceptos del objeto, Kant define dos especies de belleza en el §I6: una belleza libre (pulchritudo vaga) y una belleza meramente adherente (pulchritudo adhaerens). Y explica al respecto: "La primera no presupone concepto alguno acerca de que lo que deba ser el objeto; la segunda presupone un tal concepto y, según él, la perfección del objeto. Las primeras se denominan bellezas (por sí existentes) de ésta o aquella cosa; la otra, en cuanto dependiente de un concepto (belleza condicionada) le es atribuida a objetos que están bajo el concepto de un fin particular" (I99I, §16, B49).

Más allá de la distinción que se realiza en este parágrafo, resulta interesante comprender de qué manera se admite la presencia de conceptos relacionados al objeto en los juicios estéticos. En el enjuiciamiento de una belleza libre el juicio de gusto es puro, mientras que en el enjuiciamiento de una belleza adherente los juicios estéticos presuponen ciertos conceptos y determinaciones del objeto. Ciertamente, no son fundamentos de determinación del juicio. Como se ha visto en secciones anteriores, un juicio estético solamente puede reposar en un principio de conformidad a fin formal entre las facultades del conocimiento. Sin embargo, sí se sostiene que ciertas determinaciones del objeto son necesarias para expresar un juicio de gusto a partir de la representación. La belleza de ciertas representaciones supone un concepto del fin que determina lo que la cosa debe ser, esto es, un concepto de su perfección. Pero Kant sostiene que la belleza concierne propiamente solo a la forma, por lo que su vinculación con conceptos impide la pureza del juicio (I99I, §I6, 
B50). Por lo tanto, de tales juicios de gusto solo se puede enunciar una belleza meramente adherente de la representación.

Del tercer momento de la "Analítica de lo bello" surgen, entonces, problemas relacionados al enjuiciamiento de objetos artísticos. Existe una lectura formalista de Kant, para la que un juicio puro de gusto se sostiene en la aprehensión de la mera forma del objeto ( $y$ no en un principio de conformidad a fin formal de las facultades de conocimiento), separada de los contenidos y conceptos que trae consigo la representación. Bajo aquella lectura formalista, es imposible emitir juicios puros de gusto a partir de obras de arte. Incluso si se excluye todo concepto y determinación del objeto, un juicio de gusto sobre una obra de arte supone un concepto de "arte" intrínseco al objeto: "we must subsume a work of art under the concept art in order to be able to say that it is a "beautiful work of art'” (Guyer 1994, 25). Cada vez que aprehendemos una obra de arte damos como supuestos ciertos conceptos en la representación, incluso un concepto tan general como el de arte (o de qué tipo de arte se trata). Las conclusiones del tercer momento parecen sugerir que solo se pueden emitir juicios de belleza adherente a partir de objetos artísticos: "Kant's account of fine art seems to suggest that all judgements of artistic beauty are 'logically conditioned', which, in turn, appears to be equivalent to claiming that all artistic beauty is merely adherent" (Allison 200I, 272).

Lo problemas que se desprenden del tercer momento concernientes al arte son tratados y resueltos por Kant en la "Deducción", específicamente en los parágrafos sobre el arte bello y el genio. Al preguntarse sobre la naturaleza de la producción artística Kant debe explicar cómo se pueden emitir juicios de gusto a partir de obras de arte. Justamente para Allison, Kant propone la teoría del genio como solución a las dificultades planteadas alrededor de la belleza artística en el tercer momento (200I, 27I). Por lo tanto, se vuelve necesario investigar si y cómo Kant supera la aparente imposibilidad de que haya enjuiciamientos de belleza libre en relación con objetos artísticos.

\section{§ 2.2. Del arte bello}

A partir de lo dicho por Kant sobre el arte bello, interesan dos definiciones, siempre en contraste con el enjuiciamiento de una belleza natural. Primero, 
se sostiene con respecto al arte bello que, en el enjuiciamiento de uno de sus productos, el sujeto debe estar consciente de que es arte y no naturaleza; y, sin embargo, "la conformidad a fin en la forma de aquél debe parecer tan libre de toda sujeción a reglas arbitrarias como si fuera un producto de la mera naturaleza” (Kant 199I, §45, B 179). Segundo, se establece la diferencia entre belleza natural y belleza artística, en donde la primera es una cosa bella, y la segunda es una bella representación de una cosa (I99I, §48, BI88).

En relación con la primera definición, Kant sostiene que cuando el sujeto juzga un objeto artístico, este debe estar consciente de que es arte. La consciencia del sujeto sobre la condición artística del objeto se establece como una precondición para juzgarlo como bello arte: "unless we were aware of it as the product of a conscious intent, we could not begin to appreciate it as art" (Allison, 200I, 275). Se vuelve necesario reconocer en el arte un propósito determinado de producir algo (un producto artístico), para poder empezar a juzgarlo como tal; a pesar de que Kant sostiene que bello es aquello que place en el mero enjuiciamiento, con respecto tanto a la belleza natural como a la belleza artística (199I, §45, B 180).

En tal definición se produce una aparente paradoja, ya que, si bien es requisito que el sujeto reconozca al objeto como arte, y no como naturaleza, también se observa que Kant exige que, en los enjuiciamientos de belleza artísticas, el objeto sea juzgado como libre de todo propósito o intención, de tal forma que parezca un producto de la naturaleza. Pero advirtamos que, cuando el autor realiza el procedimiento inverso y declara que la naturaleza es bella cuando a la vez tiene viso de arte (199I, §45, BI79), la paradoja desaparece. Porque declarar que la naturaleza es bella cuando tiene viso de arte significa otorgarle una cierta conformidad a fin en ella misma indiferente para poder juzgarla estéticamente: "In fact, the requirement that such beauty look like art amounts to nothing more than that it exhibit the form of purposiveness, which certainly can be understood independently of any appeal to conscious intent" (Allison 200I, 275).

Siguiendo lo que sostiene Allison, reconocer un objeto como arte para poder juzgarlo como tal y que, sin embargo, se muestre en el juicio como un producto meramente natural, no significa que el arte deba ser una mera copia o representación de la naturaleza. Por el contrario, para poder emitir un juicio 
de gusto de un objeto artístico, es necesario aprehender el objeto como si no hubiese ningún propósito o intención en su diseño: "It is, rather, that it must seem natural in the sense of being spontaneous, unstudied, or, as Kant puts it, "unintentional", that is, as if it were a product of mere nature rather than art" (Allison 200I, 276).

Sin embargo, esto no significa que el objeto artístico deba prescindir de todo diseño o regla. Porque para poder emitir un juicio de belleza artística, la aprehensión del objeto (o su representación) debe hacer posible una relación armónica entre las facultades del conocimiento, cosa que un mal diseño del objeto podría no suscitar: "In order to be judged beautiful, the object must be subjectively purposive for judgment; that is, it must occasion a harmony of the faculties in free play, and this could not occur if it were badly designed" (Allison 200I, 276). Por lo tanto, se establece la necesidad de ciertas reglas en la producción artística a partir de tal precisión. En conclusión, en los enjuiciamientos puros de belleza artística una representación artística debería suscitar placer libremente, a pesar de los conceptos contenidos en ella.

Por otro lado, Allison desarrolla la diferencia entre belleza natural y belleza artística, en el sentido en que Kant presenta a la primera como una cosa bella y a la segunda como la bella representación de una cosa. Centra su análisis en el siguiente pasaje: "cuando el objeto es dado como producto del arte y debe ser, en cuanto tal declarado bello, tiene primeramente que ponerse por fundamento un concepto de qué deba ser la cosa” (199I, §48, B।88). Si se sigue tal lineamiento, Kant parece establecer que no pueden haber enjuiciamientos puros de belleza artística, ya que "representar algo" significa, en un sentido, "presuponer un concepto de lo que deba ser la cosa" (Allison 200I, 29I). Bajo esta definición, Kant sostendría que todos los juicios de belleza artística son juicios de belleza adherente, al ser esta la representación de una cosa y no la cosa en sí. Sin embargo, al remitirnos al tercer momento, Kant presenta tanto objetos de la naturaleza como obras de arte como ejemplos de belleza libre: "Muchas aves [...], una multitud de crustáceos del mar son, por sí, bellezas, que no convienen a ningún objeto determinado por conceptos con vistas a un fin, sino que placen libremente y por sí mismos. Así, los dibujos à la grecque, las follajerías de los marcos o de los papeles de tapizar no significan nada en sí mismos; nada representan, ningún objeto bajo un concepto determinado, y son bellezas libres" (199I, §I6, B49). 
Kant realiza el mismo procedimiento a la hora de presentar ejemplos de belleza adherente: "la belleza de un hombre (...), la belleza de un caballo, de un edificio (...) supone un concepto del fin que determina lo que la cosa debe ser, y en consecuencia, un concepto de su perfección, y es entonces sólo una belleza adherente" (199I, §16, B50). El autor ha asumido desde un principio que tanto objetos artísticos como naturales pueden representar bellezas libres o bellezas adherentes.

Para explicar de qué forma se admiten los juicios puros de gusto con respecto a la belleza artística, Allison propone identificar los diferentes significados de "representación" que Kant utiliza aleatoriamente a lo largo de las secciones aquí analizadas. Allison busca en el texto un significado de "representación" que se aplique únicamente a los objetos artísticos y no comprometa a toda belleza artística como belleza adherente.

En primer lugar, Allison repara en el siguiente argumento, en donde Kant sostiene que la bella representación de un objeto "es propiamente sólo la forma de la presentación de un concepto, a través de la cual éste es comunicado universalmente" (I99I, §48, B190). Aquí, una "bella representación" es entendida como la expresión de ideas estéticas. Estas son definidas como representaciones de la imaginación que dan a ocasión a múltiples reflexiones, sin que se les pueda determinar bajo ningún concepto (I99I, §49, B|93). Por un lado, las ideas estéticas tienden hacia algo que yace fuera del límite de la experiencia y buscan aproximarse a conceptos de la razón, lo que les da la apariencia de una realidad objetiva; por el otro, debido a que son intuiciones internas de los objetos artísticos, ningún concepto puede serles enteramente adecuado (199I, §49, B 194). Son presentadas a partir de una facultad propia del genio: el espíritu. Sería posible emitir juicios de gusto sobre belleza artística a partir de la aprehensión de la representación en la cual ya está dada una idea estética, que es producto de la imaginación en su libertad y suscita una relación entre esta y el entendimiento. Sin embargo, las ideas estéticas también están presentes en la belleza natural y, por lo tanto, no se aplican únicamente a la belleza artística.

Además, Allison presenta otra definición de lo que Kant entiende por "representación”. Cuando este indica que en los enjuiciamientos de belleza artística "tiene primeramente que ponerse por fundamento un concepto de qué deba 
ser la cosa" (I99I, §48, I88), se refiere principalmente a qué forma o género de arte pertenece: "This phrase [that in order to judge the beauty of a work of art it is necessary to have a concept of the "kind of thing the object is meant to be'] refers primarily to the artform or genre of a work. (...) Otherwise expressed, it indicates the kind of work that it must be seen as being" (Allison 200I, 295). En la lectura de Allison, "lo que la cosa debe ser" significa que la obra representa el tipo de trabajo que es. Allison sostiene que este es un sentido ejemplificativo de representación, en el que cada obra es ejemplo del tipo de arte al que pertenece. Además, resalta que existe una diferencia entre lo ejemplificado en la representación en tal pasaje, y lo ejemplificado en los casos de belleza adherente presentados por Kant: "There [in cases of adherent beauty] it was a matter of some extra-aesthetic purpose that the work was intended to subserve, and that imposed extra-aesthetic constraints on what is appropriate. Here [in an exemplative sense of representation], by contrast, what is exemplified, and therefore represented, is just the artform or genre itself, the kind of work it is seen as being by its audience" (Allison 200I, 296).

Con esto, Allison encuentra un sentido de representación que es aplicable a todas las obras de arte bello, y no a la belleza natural, debido a que se deriva de un análisis de la naturaleza de tal arte (Allison, 200I, 296). Además, aquí se refuerza la idea de que, ante una obra de arte, el sujeto debe reconocerla como tal para poder emitir un enjuiciamiento de belleza artística. Sin embargo, tal definición no termina de resolver el problema planteado, debido a que el requisito de que la apreciación de belleza artística presuponga un concepto de lo que la obra deba ser continúa determinando todo juicio de belleza artística como meramente adherente. Por lo tanto, Allison propone reformular el problema de la distinción entre juicios de belleza libre y juicios de belleza adherente a la luz de la concepción de ideas estéticas (Allison, 200I, 297), como explicaremos en la siguiente sección.

\section{§ 2.3. Sobre el genio}

Queda por determinar de qué modo el genio puede ser una solución a los problemas desarrollados que se desprenden del tercer momento. Primero, es necesario resolver en qué sentido Kant declara que el arte bello, al mismo tiempo que es reconocido como arte, debe parecer tan libre de toda sujeción 
a reglas como si fuese un producto natural. En un primer momento, Kant define al genio de la siguiente manera: "Genio es el talento (don natural), que le da la regla al arte. Dado que el talento, como facultad productiva innata del artista, pertenece, él mismo, a la naturaleza, podría uno entonces expresarse también así: genio es la innata disposición del ánimo (ingenium) a través de la cual la naturaleza le da la regla al arte” (199I, §46, BI8I).

Kant declara que la naturaleza le da la regla al arte a través del genio, una innata disposición del ánimo en el sujeto que produce arte bello. Después del desarrollo del primer problema, queda claro que todo arte, para ser juzgado como tal, supone reglas, a partir de las cuales viene primeramente a ser representado como posible un producto artístico (I99I, §46, BI8I). Pero también se establece que el concepto de bello arte no permite que el juicio sobre la belleza de un producto artístico sea derivado de una regla que tenga un concepto como fundamento. El arte bello tampoco puede inventarse la regla para la producción de objetos bellos. Sin embargo, debido a que un producto sin regla precedente no puede llamarse arte, Kant argumenta que "tiene la naturaleza que dar la regla al arte en el sujeto ( $y$ a través del temple de las facultades de éste); es decir, el arte bello es solo posible como producto del genio" (199I, §46, BI82). Por lo tanto, sostiene que las bellas artes deben ser consideradas como artes del genio.

De lo anterior se desprende la primera definición de genio. Primero, el genio es un talento para la producción de objetos (artísticos) sobre los que no se puede dar ninguna regla determinada. Se le otorga una propiedad de originalidad al genio, ya que él le prescribe la regla al arte. Segundo, los productos del genio deben ser ejemplares; deben servir como criterio o regla de enjuiciamiento. Tercero, no puede dar razón de cómo produce un objeto artístico, sino que "da la regla en cuanto naturaleza, y de ahí que el propio autor de un producto (...) no sepa cómo se encuentran en él las ideas para ello” (199I, §46, BI82). Las reglas de producción artística no se fundamentan en conceptos, sino que la naturaleza le da la regla al arte por medio de la actividad del genio. Por último, "la naturaleza, a través del genio, prescribe la regla no a la ciencia, sino al arte; e incluso ello solamente en la medida en que este último deba ser bello arte" (199I, §46, BI82). 
Por lo tanto, en el §46 el genio es visto como un "preferido de la naturaleza" que le prescribe una regla al arte bello para establecer las posibilidades de su producción. El genio es alguien a quien la naturaleza ha dotado para la producción del bello arte. Las bellas artes, como artes del genio, pueden parecer "meros productos de la naturaleza" en tanto son productos de la naturaleza del artista: "such art can seem like nature, even though we are conscious of it as art, precisely because it is a product of nature (the nature of the artist)" (Allison 200I, 279). La originalidad se vuelve la propiedad más importante del genio, ya que produce algo para lo que ninguna regla determinada puede estar dada, por lo que no puede producir un objeto artístico siguiendo un conjunto de reglas. A partir de estas deducciones, el bello arte puede ser visto como libre de propósito o intención, a pesar de que ha habido una cierta intencionalidad en su diseño que puede ser reconocida.

Por otro lado, interesa desarrollar el §49, concerniente a las facultades del ánimo que constituyen al genio, en donde se brinda una segunda definición del término, a partir de las precisiones hechas sobre el espíritu y las ideas estéticas (definidas en la sección anterior). En esta sección, nos centraremos en dos aspectos del genio. Primero, Kant establece que el genio es un talento para el arte en el que reglas nítidamente conocidas tienen que ser precedentes y determinar el procedimiento de su producción (199I, §49, B 199). Luego, Kant sostiene que es un talento que se muestra en la exposición o expresión de ideas estéticas, que hacen representable a la imaginación en su libertad (1991, $\S 49, \mathrm{~B}$ 199). En ese sentido, la imaginación procede independiente de toda dirección por reglas; $y$ sin embargo, se presenta como conforme a fin para la presentación de objetos dados. En ese sentido, el genio establece una relación armónica entre sus facultades de conocimiento, en donde la imaginación en su libre juego presenta ideas estéticas al entendimiento para la producción de arte bello: “(...) el genio consiste, entonces, propiamente, en la feliz relación que ninguna ciencia puede enseñar y ninguna laboriosidad aprender, de descubrir ideas para un concepto dado y, por otra parte, encontrar la expresión para ellas a través de la cual puede ser comunicado a otros el temple subjetivo del ánimo por ese medio efectuado, como acompañamiento de un concepto. (...) expresar lo innominable en el estado del ánimo a propósito de una cierta representación y hacerlo universalmente comunicable -consista la expresión en lenguaje, pintura o plástica-, exige una potencia para aprehender el juego 
de la imaginación que pasa velozmente y para unificar en un concepto (...), el cual puede comunicarse sin la coartación de reglas" (I991, §49, B।98-199).

Como se ha dicho antes, Allison se propone resolver la división entre belleza libre y belleza adherente a partir de la concepción de ideas estéticas. Realiza este procedimiento evaluando la división kantiana de las bellas artes y las diferencias que resalta Kant entre el arte escultórico y el arte arquitectónico. Kant los distingue de la siguiente manera: "El primero es aquel que presenta corpóreamente conceptos de cosas, tal como éstas podrían existir en la naturaleza (pero, como arte bello, atendiendo a la conformidad a fin estética); el segundo es el arte de presentar a tal propósito, pero también a la vez en conformidad a fin, conceptos de cosas que sólo son posibles a través del arte y cuya forma no tiene por fundamento de determinación a la naturaleza, sino un fin arbitrario. En el último lo capital es un cierto uso del objeto artístico, a lo cual son limitadas, como a su condición, las ideas estéticas” (I99I, §5I, B207-B208).

Además, declara que la intención principal de la escultura es "la mera expresión de ideas estéticas” (199I, §5I, B208). Según Allison, Kant considera la belleza del arte escultórico como una belleza libre, debido a que sólo tiene como propósito expresar las ideas estéticas del genio. Estas no son propiamente conceptos; por el contrario, ningún concepto puede serles enteramente adecuado. Además, están contenidas en la aprehensión de la forma, por lo que es posible realizar juicios puros de belleza estética y aprehender, por medio de la reflexión, las ideas estéticas presentadas por el genio.

En cambio, el propio Allison observa que, si bien la arquitectura también permite presentar ideas estéticas, sus obras tienen un propósito extra estético. Presuponen un cierto uso o función: "the aesthetic evaluation of a work of architecture is subject to extra-aesthetic constraints, stemming from its intended function" (200I, 298). En la lectura de Allison, se vuelve esencial que no se reconozca un propósito o intencionalidad objetiva, ajena a la presentación de ideas estéticas en el objeto, esto es, que plazca únicamente por sí mismo: "What is essential is that it is not meant to serve any extrinsic purpose, that it is liked purely for itself, in virtue of the aesthetic ideas it expresses" (200I, 298). 
Las ideas estéticas no impiden la posibilidad de emitir juicios puros de belleza artística. Estas no se adecúan a conceptos o reglas determinadas y solo se presentan como contenidos objetivos en tanto se aproximan a conceptos de la razón. La aprehensión de ideas estéticas en el juicio suscita la reflexión del sujeto y activa una relación armónica de sus facultades de conocimiento, lo que permite la sociable comunicación del juicio: "Arte bello es (...) un modo de representación que es en sí mismo conforme a fin y que, aun carente de fin, promueve la cultura de las fuerzas del ánimo con vistas a la sociable comunicación” (Kant, 199I, §44, BI79).

\section{§ 3. La jardinería de placer en el Siglo XVIII}

\section{§ 3.1. La jardinería de placer dentro de la clasificación de las artes}

Durante el Siglo XVIII surgió una discusión alrededor de la jardinería como arte que implicaba un debate entre formas distintas de pensar y manipular la naturaleza: la tradición de jardinería francesa y la tradición de jardinería inglesa. Con posiciones distintas y, más que todo, enfrentadas (la tradición inglesa surge en respuesta al radical formalismo francés), en el jardín francés la naturaleza era sometida, modifica y domesticada a voluntad por el hombre, mientras que en el jardín inglés se buscaba representar a la naturaleza en su total libertad.

Para Kant, la jardinería de placer es una forma de producción artística. El filósofo la incluye dentro de su proyecto de división de las bellas artes y la agrupa dentro de las artes plásticas o artes de expresión de ideas en la intuición de los sentidos, que a su vez son divididas en artes de la verdad de los sentidos y en artes de la apariencia de los sentidos (199I, §5I, B207). Llama a la primera plástica, y a la segunda pintura o arte pictórico: "Ambas hacen de figuras en el espacio expresión para ideas: aquéllas hace figuras conocibles para dos sentidos, la vista y el tacto (si bien éste no con designio de belleza), ésta solo para el primero" (199I, §5I, B207).

La jardinería de placer pertenece a las artes pictóricas, junto con la pintura: "El arte pictórico, en cuanto segunda especie de arte figurativo que presenta la apariencia de los sentidos artísticamente enlazada con ideas, lo dividiría yo 
en la bella descripción de la naturaleza y en el de la bella combinación de sus productos" (Kant 199I, §5I, B208). La primera especie de belleza es propia de la pintura; mientras que la segunda, de la jardinería de placer. La pintura solo da la apariencia de la extensión corpórea, mientras que la jardinería: “(...) da (...) (la extensión corpórea) de acuerdo a la verdad, mas sólo la apariencia de utilización y empleo para otros fines que no sean el juego de la imaginación en la contemplación de sus formas. La última [la jardinería] no es otra cosa que el ornato del suelo con la misma diversidad (pastos, flores, arbusto y árboles, y aun aguas, colinas y valles) con que la naturaleza lo presenta a la mirada, sólo que compuestas de otra suerte y en conformidad con ciertas ideas. La bella composición de cosas corpóreas, sin embargo sólo se ofrecen a los ojos, como la pintura (...) (Kant 199I, §5I, B209-210)”.

Para Kant, el placer estético en la jardinería de placer se suscita gracias a las ideas estéticas presentadas por el genio en la mera contemplación de la composición de los elementos naturales. Por ello, el debate entre la tradición francesa y la tradición inglesa resulta útil para entender su posición respecto de la importancia del genio en la producción de arte bello y la posibilidad de emitir juicios puros de belleza artística a partir de la aprehensión de las ideas artísticas propuestas por él.

\section{§ 3.2. La tradición formal francesa}

El jardin à la française fue el modelo predominante de diseño de jardines durante el Siglo XVII. Se inspiró principalmente en el Renacimiento Italiano, del cual tomó numerosos principios para aplicarlos al diseño del espacio. Su base principal fue la geometría, como elemento rector de todo el proceso creativo. En un jardín francés la naturaleza era sometida y domesticada al diseño que le aplicaba el jardinero. La regularidad y perfección representada en el espacio le era impuesta a la naturaleza, en un lugar en donde la voluntad del hombre era rectora. Claramente, un jardín francés debe ser recorrido y apreciado como una obra de arte.

André Le Nôtre es el máximo exponente de la tradición de jardinería francesa. Sus obras maestras son los jardines de Vaux-Le-Vicomte y del palacio de Versailles. Basta leer una descripción de Vaux-Le-Vicomte para encontrar las 
principales ideas de la tradición francesa condensadas en un solo espacio: "El palacio residencial, el château (...) se halla inscrito como parte de ese recorrido longitudinal, señala la transición entre el exterior, desde la entrada del patio, hacia la inmersión gradual en un mundo, el del jardín, organizado de manera que la naturaleza vaya siendo domesticada según los dictados de la geometría racional de las calles rectilíneas y las plazas estrelladas, por medio de parterres, fuentes de agua, estanques, canales, caminos transversales, bosquetes, que hacen presente al paseante la idea de que hay un dentro y un fuera del jardín, una frontera marcada entre la naturaleza recreada y mejorada del interior, y la naturaleza salvaje del exterior, del resto del mundo natural no afectado todavía por el trabajo del jardinero redentor” (Rodríguez Llera 2009, 77).

Para la jardinería francesa, el genio calza como el autor de un producto de arte. La actividad del genio se ve explicitada en la obra de arte, en la que se aprecia claramente una cierta intencionalidad para su producción. El jardinero francés le prescribía una regla al espacio, y tal intervención quedaba expresada en el diseño distintivo del jardín francés.

Ciertamente, Kant se mostraba reacio ante toda obra de arte cuyo diseño se fundamentara sólo en una cierta regularidad, debido a que, para él, tiene escasa capacidad de producir en el sujeto la relación armónica entre sus facultades de conocimiento necesaria para producir juicios de gusto: "Todo lo rígido-regular (que se aproxima a la regularidad matemática) conlleva en sí algo contrario a gusto: es que no brinda ningún entretenimiento prolongado con su contemplación, sino que aburre, en la medida en que no tenga expresamente por propósito el conocimiento o un fin práctico determinado"(I99|, $\S 22$, B7I). Para Kant, la regularidad compulsiva de los jardines franceses no puede activar la facultad de imaginación en su libertad. Sin embargo, como se ha demostrado, es posible producir juicios puros de belleza artística en la contemplación del objeto a partir de la aprehensión de las ideas estéticas contenidos en este. La capacidad de emitir juicios puros de los jardines franceses significaría que la representación placería libremente a pesar de los conceptos contenidos en ella. 


\section{§ 3.3. La tradición inglesa}

Es importante reparar en que la tradición inglesa surge como respuesta y rechazo a la regularidad y modificación indiscriminada de la naturaleza de la que se jactaba la tradición formal francesa. Surge de un cambio de mirada radical sobre la concepción de la belleza natural. En ella, la naturaleza no está sometida a la voluntad del hombre, sino que ella es maestra y regla. En un jardín inglés la naturaleza "se diseña a sí misma".

En el jardín inglés el espacio "pintoresco" es una idea predominante: "Alrededor de 1770 lo pintoresco comenzó a formar parte del proyecto de paisaje. Con lo que se puso de moda un nuevo jardín salvaje, con árboles caídos y hondonadas cubiertas de helechos, dispuesto todo como si se tratase de un paisaje natural de características geológicas espectaculares, con escarpes y barrancos" (Steenbergen 200I, 26I). Los elementos eran combinados de tal forma en el espacio que cada vista generada en el jardín podía apreciarse a manera de una pintura. La idea de un paisaje pintoresco dentro de la tradición inglesa estaba íntimamente ligada a la concepción de un espacio lo más "natural" posible, en donde la naturaleza se diseña a sí misma: "La naturaleza, expresándose a sí misma, carece de tendencias ortogonales, y ningún lugar hace uso de reglas regulares para componerse bien. El reflejo correcto sigue sus pautas, pues en eso la naturaleza es maestra. La perspectiva específica del punto de paisaje encaja valles y colina, ríos y nubes, praderas y bosques según su natural ser. (...) el pintor ha de saber añadir luz y color como aglutinante de los elementos. Y prácticamente nada más. A no ser que quiera agregar a ese paisaje natural las imágenes de la vida y de la cultura, animales, hombre y arquitectura básicamente" (Rodríguez Llera 2009, 95-96).

William Kent, pintor y jardinero inglés, “(...) formuló el trazado del jardín en un estilo de transición de la geometría regular hacia la propuesta de un nuevo tipo de ajardinamiento irregular de inspiración pictórica, basado en la creación de efectos sobre equilibrios compositivos asimétricos, de vistas en ángulo, de escenas calificadas de "pintorescas” (Rodríguez Llera 2009, 90). Las obras de jardinería inglesa se centraron en la autorrepresentación de la naturaleza como sistema irregular, en la cual esta se mostraba en un estado silvestre y lo más salvaje posible, pero mejoradas por la participación imperceptible del jardinero. 
El resultado es un espacio que, como obra de arte, tiene la apariencia de naturaleza, pero al mismo tiempo es naturaleza. El genio del jardín inglés es un jardinero apenas reconocible en el diseño de su obra. Ciertamente, es como si la naturaleza "se diera la regla a sí misma". El objetivo de un jardinero inglés no era exaltar su intervención en el espacio. Por el contrario, al mantenerse relativamente al margen, se buscaba comprender a la naturaleza en su total libertad. Para Kant, la contemplación de un jardín inglés impulsaba la libertad de la imaginación, justamente porque la presentación de ideas estéticas dentro de la obra artística se centraba en el reconocimiento de un espacio aparentemente libre de reglas de producción y, en ese sentido, natural.

\section{§ 4. Conclusión}

La dificultad señalada en el tercer momento de la "Analítica" puede considerarse superada. La imposibilidad de emitir juicios puros de belleza artística, debido a que las obras de arte presuponen un concepto de arte intrínseco a ellas, termina por resolverse en los pasajes sobre el arte bello y el genio. El genio se propone como posible solución al problema de la categorización de toda belleza artística como mera belleza adherente.

Siguiendo a Allison, podemos admitir juicios puros de belleza artística a partir de la concepción de ideas estéticas. Estas son productos de la imaginación en su libertad; pero para que se suscite la reflexión, para que tenga lugar un juicio reflexionante, es necesario un entendimiento que legisle sobre tales presentaciones. El genio es capaz de lograr una relación armónica entre las facultades de conocimiento; presenta gracias a su espíritu las ideas estéticas necesarias en la obra de arte. La aprehensión de la forma trae consigo los contenidos de la obra, esto es, las ideas estéticas del genio. En una intuición, la obra de arte, están contenidas las ideas estéticas.

A partir de los ejemplos, queda claro cómo es posible emitir juicios puros de gusto de belleza artística, a pesar de que se traten de expresiones radicalmente distintas de un mismo género artístico. Ambas formas contienen ideas estéticas presentadas por el genio artístico, cuya aprehensión en la contemplación de la obra abre la posibilidad de emitir un juicio estético puro 
de ambas expresiones artísticas. Claramente Kant se inclina por la aparente espontaneidad y libertad de los jardines ingleses. Sin embargo, la contemplación de un jardín francés también puede suscitar un sentimiento de placer en el sujeto, si no fundamenta su juicio en los conceptos presentados en la obra. En todo caso, es importante notar que, al fundamentar un juicio puro de gusto sobre el principio de conformidad a fin formal, esto no significa que no haya ideas estéticas en la obra. Por el contrario, su importancia radica en que ellas admiten la reflexión y discusión sobre la obra de arte que se juzga.

\section{Bibliografía}

Albers, Lucia, 1991. "The perception of Gardening as Art”. Garden History 19 (2), 163 174. http://www.jstor.org/stable/I586892. Consultado 2 de julio de 2016.

Allison, Henry, 200I. Kant's Theory of Taste: a Reading of the Critique of Aesthetic Judgment. New York: Cambridge University Press. https://doi.org/I0.1017/CBO978051/61267I

Baridon, Michel, 2008. Los jardines. Paisajistas, Jardineros, Poetas (Siglos XVIII-XX). Traducción de Juan Calatrava y José Luis López Jiménez. Madrid: Abada Editores.

Bending, Stephen, 1992. "Re-Reading the Eighteenth-Century English Landscape Garden”. Huntington Library Quarterly 55 (3), 379-399. http://www.jstor.org/stable/3817684. Consultado 2 de julio de 2016. https://doi.org/10.2307/38I7684

Brook, Isis, 2008. "Wildness in the English Garden Tradition: A Reassessment of the Picturesque from Environmental Philosophy". Ethics and the Environment I3 (I), 105 119. http://www.jstor.org/stable/40339150. Consultado 2 de julio de 2016. https://doi. org/10.2979/ete.2008.13.1.105

Cooper, David, 2006. A Philosophy of Gardens. New York: Oxford University Press.

Dispinzio, Joseph, 200I. "From Garden to Landscape:Jean-Marie Morel and the Transformation of Garden Design". AA Files 44, 6-20. http://www.jstor.org/stable/29544230. Consultado 2 de julio de 2016.

Fariello, Francesco, 2004. La arquitectura de los jardines. De la Antigüedad al siglo XX. Traducción y edición de Jorge Sainz. Barcelona: Editorial Reverté.

Ferrari, G. R. F., 2010. "The Meaninglessness of Gardens". The Journal of Aesthetics and Art Criticism 68 (I), 33- 45. http://www.jstor.org/stable/25622I2I. Consultado: 2 de Julio del 2016. https://doi.org/I0.1III/j.1540-6245.2009.01390.x 
Guyer, Paul, 1994. "Kant's Conception of Fine Art”. The Journal of Aesthetics and Art Criticism 52 (3), 275-285. http://www.jstor.org/stable/43|427. Consultado 4 de julio del 2016. https://doi.org/10.2307/43/427

- 1997. "Jupiter's Eagle to Warhol's Boxes: The Concept of Art from Kant to Danto". Philosophical Tropics 25 (I), 83-II5. http://www.jstor.org/stable/43I5425I. Consultado 5 de Julio del 2016. https://doi.org/I0.5840/philtopics 199725120

Kant, Immanuel, 199I. Crítica de la facultad de juzgar. Traducción de Pablo Oyarzún. Caracas: Monte Ávila.

Lenabat, Marianne, 2009. "From the pepper garden to the botanical garden: Kant on the appreciation of natural beauty". Postgraduate Journal of Aesthetics 6 (3), 25-39.

Levi, Albert, 1984. "Nature and Art". The Journal of Aesthetic Education I8 (3), 5-2I. http://www.jstor.org/stable/3332672. Consultado 2 de julio de 2016.

López, Antonio y Ángel Ramos, 200I. Valoración del paisaje natural. Madrid: Abada.

Miller, Elaine, 2002. The Vegetative Soul: From Philosophy of Nature to Subjectivity in the Feminine. New York: State University of New York Press.

Rodríguez Llera, Ramón, 2009. Paisajes arquitectónicos: lo regular como norma, lo irregular como sistema. Valladolid: Universidad de Valladolid, Secretariado de Publicaciones e Intercambio Editorial.

Ross, Stephanie, 1987. “The Picturesque: An Eighteenth-Century Debate”. The Journal of Aesthetics and Art Criticism 46 (2), 27I-279. http://www.jstor.org/stable/43I865. Consultado 2 de julio de 2016.

Steenbergen, Clemens y Wouter Reh, 200I. Arquitectura y paisaje. La proyectación de los grandes jardines europeos. Barcelona: Editorial Gustavo Gili.

Townsend, Dabney, 1997. "The Picturesque". The Journal of Aesthetics and Art Criticism 55 (4), 365-376. http://www.jstor.org/stable/430924. Consultado el 02 de Julio del 2016. 